\title{
DETERMINING SOLUTIONS OF FUZZY CELLULAR NEURAL NETWORKS WITH FLUCTUATING DELAYS
}

\author{
Ivan P. Stanimirović
}

\begin{abstract}
This paper deals with the problem of nonperiodic arrangements for fuzzy cell neural systems with fluctuating delays. By utilizing compression mapping and Krasnoselski's settled point hypothesis and developing some appropriate Lyapunov functionals, adequate conditions are set up for the presence and worldwide exponential solidness of solutions of FCNNs with fluctuating delays. In addition, illustrative examples are set up to exhibit a model.
\end{abstract}

Keywords. Cellular neural networks; fuzzy; fluctuating delays; nonperiodic solutions.

\section{Introduction}

Celluar neural nets (CNNs), initially presented in [1], have pulled in much consideration lately. This is generally on the grounds that they have the extensive variety of promising applications in the fields of related memory, parallel figuring, design acknowledgment, flag handling and streamlining. CNNs are portrayed by essential circuit units called cells. Every unit forms a few information flags and delivers a yield flag which is gotten by different units associated with it including itself.

In the execution of a flag or impact going through neural systems, time delays do exist and influence dynamical behavior of a working neural network. As of late there have been a few outcomes about dynamical practices of deferred neural systems including worldwide exponential steadiness of balance focuses, intermittent and relatively occasional arrangements [2, 3].

Other than defer impacts, it has been seen that numerous transformative procedures, including those identified with neural systems, may display incautious impacts. In these developmental procedures, the arrangements of framework are not consistent but rather present hops which can cause shakiness of dynamical frameworks. Thus, numerous neural systems with motivations have been contemplated broadly, and a lot of writing are engaged on the issue of the presence and steadiness

Received January 21, 2019; accepted February 05, 2019

2010 Mathematics Subject Classification. Primary 62M45; Secondary 68T99 
of a balance point [4]. The presence and dependability of periodic solution of neural network with impulses are researched extensively by many authors [5, 6].

In [7], another compose cell neural systems display called fuzzy cell neural systems (FCNNs) is introduced. FCNNs joined fuzzy task with cell neural systems.

In any case, it is important that Takagi-Sugeno (T-S) fuzzy neural systems are not quite the same as FCNNs. T-S fuzzy neural systems depend on an arrangement of fuzzy guidelines to depict nonlinear framework. As of late analysts have discovered that FCNNs are helpful in picture preparing, and many fascinating outcomes have been introduced on steadiness of FCNNs. For instance, in [8], applying straight network imbalance (LMI) approach, contemplated presence, uniqueness and worldwide asymptotic steadiness of fuzzy cell neural systems with asymptotic relentlessness of cushioned cell neural frameworks with spillage delay under imprudent annoyances. The authors in [9] acquired the outcomes of asymptotic steadiness for fuzzy cell neural systems with time-shifting postponements. In [10], the steadiness of fuzzy cell neural systems is examined with time-changing delay in spillage term without accepting the boundedness of initiation function. Other related works readers can refer to [11].

However, in applied sciences, the existence of nonperiodic arrangements assumes a key job in portraying the conduct of nonlinear differential conditions. For instance, hostile to intermittent trigonometric polynomials are vital for the investigation of addition issues, against occasional wavelets and simple voltage transmission are frequently against intermittent process, in this way it is profitable to consider nonperiodic solutions. Meanwhile, anti-periodic solution, as a special case of periodic solution, has an important research value in dynamic behavior of the neural networks. In recent years, the problem of nonperiodic solution of CNNs, Hopfield neural nets and recurrent neural nets has been studied by many scholars (see [12, 13, 14] and references therein). For example, in [12], the author studied the presence and exponential security of the counter occasional arrangements of intermittent neural systems with time-differing and persistent dispersed deferrals. In [13], applying imbalance procedure and dependent on Lyapunov practical hypothesis, the authors examined the presence and worldwide exponential security of against intermittent answer for defer CNNs with hasty impacts. In any case, to the best of our insight, there are not very many outcomes on the issues of against occasional answers for fuzzy cell neural systems (FCNNs) with fluctuating delays and hasty impacts.

It is reasonable to proceed the examination of the presence and stability of nonperiodic arrangements for FCNNs with period-varying delays and impulsive effects. Here, we are concerned with the next model: 


$$
\left\{\begin{aligned}
x_{i}^{\prime}(t) \quad & -a_{i}(t) x_{i}(t)+\sum_{j=1}^{n} d_{i j}(t) f_{j}\left(x_{j}(t)\right) \\
& +\bigwedge_{j=1}^{n} a_{i j}(t) g_{j}\left(x_{j}\left(t-t_{i j}(t)\right)\right) \\
& +\bigvee_{j=1}^{n} b_{i j}(t) g_{j}\left(x_{j}\left(t-t_{i j}(t)\right)\right) \\
& \left.+E_{i}(t)\right], t \geq 0, t \neq t_{k}, k \in N^{+}, \\
\Delta\left(x_{i}\left(t_{k}\right)\right)= & x_{i}\left(t_{k}^{+}\right)-x_{i}\left(t_{k}^{-}\right)=I_{i k}\left(t_{k}, x_{i}\left(t_{k}\right)\right), \\
x_{i}(t) \quad & \varrho_{i}(t), t \in[-t, 0], i=1,2, \cdots, n .
\end{aligned}\right.
$$

where $n$ is the amount of elements in the net. $x_{i}(t)$ is the activations of the $i$ th neuron at the time $t . a_{i}(t), d_{i j}(t), a_{i j}(t), b_{i j}(t), E_{i}(t), f_{j}(t), g_{j}(t), t_{i j}(t)$ are continuous functions on $R . \quad a_{i}(t)>0$ represents the amplification function. $d_{i j}(t)$ denotes the synaptic connection weight of the unit $j$ on the unit $i$ at time $t$. Thus, $a_{i j}(t)$ and $b_{i j}(t)$ are elements of fuzzy feedback MIN and MAX template, correspondingly. $\Lambda$ and $\bigvee$ represent the fuzzy AND and OR operation, correspondingly. $E_{i}(t)$ denotes the $i$-th component of an external input source introduced from outside the network to the $i$ th cell. $t_{i j}(t)$ is time-varying delay satisfying $0 \leq t_{i j}(t) \leq t, t$ is a positive constant. $f_{j}(\cdot)$ and $g_{j}(\cdot)$ are the activation functions. $\Delta x_{i}\left(t_{k}\right)=x_{i}\left(t_{k}^{+}\right)-x_{i}\left(t_{k}^{-}\right), x_{i}\left(t_{k}^{+}\right)=\lim _{h \rightarrow 0+} x_{i}\left(t_{k}+h\right), x_{i}\left(t_{k}^{-}\right)=$ $\lim _{h \rightarrow 0-} x_{i}\left(t_{k}+h\right),(i=1,2, \cdots, n, k=1,2, \cdots) .\left\{t_{k}\right\}$ is a sequence of real numbers such that $t_{1}<t_{2}<\cdots$ and $\lim _{k \rightarrow+\infty} t_{k}=+\infty$.

The primary motivation behind this paper is to think about the presence and worldwide exponential solidness of hostile to occasional arrangements of (1).

The framework of this paper is as per the following. In Sect. 2, we present a few definitions and lemmas. In Sect. 3, we set up new adequate conditions for the presence of the counter occasional arrangements of framework (1). In Faction 4, by building reasonable Lyapunov practical, we infer adequate conditions for the worldwide exponential strength of hostile to intermittent arrangements of framework (1). A numerical model is given to demonstrate the adequacy of our outcomes in Sect. 5. At last a general end is attracted Sect. 6 .

\section{Preliminaries}

Let us present the following:

$$
\begin{aligned}
& a_{i}^{-}=\min _{t \in[0, \omega]}\left|a_{i}(t)\right|, a^{+}=\max _{1 \leqslant i \leqslant n} \max _{t \in[0, \omega]}\left|a_{i}(t)\right|, \\
& \bar{d}_{i j}=\max _{t \in[0, \omega]}\left|d_{i j}(t)\right|, \bar{d}=\max _{1 \leqslant i \leqslant n} \max _{t \in[0, \omega]}\left|d_{i j}(t)\right|,
\end{aligned}
$$




$$
\begin{aligned}
& \bar{a}_{i j}=\max _{t \in[0, \omega]}\left|a_{i j}(t)\right|, \bar{a}=\max _{1 \leqslant i \leqslant n} \max _{t \in[0, \omega]}\left|a_{i j}(t)\right|, \\
& \bar{b}_{i j}=\max _{t \in[0, \omega]}\left|b_{i j}(t)\right|, \bar{b}=\max _{1 \leqslant i \leqslant n} \max _{t \in[0, \omega]}\left|b_{i j}(t)\right|, \\
& \bar{E}=\max _{1 \leqslant i \leqslant n} \max _{t \in[0, \omega]}\left|E_{i}(t)\right|, \chi_{i}=e^{\int_{0}^{\omega} a_{i}(\phi) d \phi} .
\end{aligned}
$$

Here, the next assumptions are made

(A1) For $i, j=1,2, \cdots, n, k=1,2, \cdots$, there exist $\omega>0$ such that for $\Omega \in R$

$$
\begin{gathered}
a_{i}(t+\omega)=a_{i}(t), t_{i j}(t+\omega)=t_{i j}(t), \\
a_{i j}(t+\omega) g_{j}(-\Omega)=-a_{i j}(t) g_{j}(\Omega), \\
b_{i j}(t+\omega) g_{j}(-\Omega)=-b_{i j}(t) g_{j}(\Omega), \\
d_{i j}(t+\omega) f_{j}(-\Omega)=-d_{i j}(t) f_{j}(\Omega), \\
E_{i}(t+\omega)=-E_{i}(t), I_{i k}(t+\omega, \Omega)=-I_{i k}(t,-\Omega) .
\end{gathered}
$$

(A2) $f_{j}(\cdot), g_{j}(\cdot) \in C(R \times R, R)$, and the nonnegative values $M_{f}, M_{g}, m_{j}, n_{j}(j=$ $1,2, \cdots, n)$ exist such that, for $u, \Omega \in R$,

$$
\begin{gathered}
f_{j}(0)=0, \quad\left|f_{j}(t, u)\right| \leqslant M_{f}, \quad\left|f_{j}(u)-f_{j}(\Omega)\right| \leqslant m_{j}|u-\Omega|, \\
g_{j}(0)=0, \quad\left|g_{j}(t, u)\right| \leqslant M_{g}, \quad\left|g_{j}(u)-g_{j}(\Omega)\right| \leqslant n_{j}|u-\Omega| .
\end{gathered}
$$

(A3) For $i, j=1,2, \cdots, n, k=1,2, \cdots$, there exists a positive integer $q$ such that

$$
I_{i(k+q)}=I_{i k}, t_{k+q}=t_{k}+\omega .
$$

(A4) For $i, j=1,2, \cdots, n, k=1,2, \cdots$, there exist $c_{i k}>0$ such that

$$
\left|I_{i k}(t, u)-I_{i k}(t, \Omega)\right| \leqslant c_{i k}|u-\Omega|, \forall t \in[0, \omega], u, \Omega \in R .
$$

Remark 2.1 In assumption (A2), the activating functions $f_{j}, g_{j}, j=1,2, \cdots, n$, are typically assumed to be bounded and Lipchtiz continuous and need not to be differential.

Consider $x(t)=\left(x_{1}(t), x_{2}(t), \cdots, x_{n}(t)\right)^{T} \in R^{n}$, whereat $T$ is the transpositioning. The starting assumptions based on (1) are determined by:

$$
x(t)=\varphi(t), \quad t \in[-t, 0],
$$

where $\varphi(t)=\left(\varphi_{1}(t), \varphi_{2}(t), \cdots, \varphi_{n}(t)\right)^{T} \in R^{n}, \varphi_{i}(i=1,2, \cdots, n)$ are continuous with norm

$$
\|\varphi\|=\sup _{t \in[-t, 0]}\left(\sum_{i=1}^{n}\left|\varphi_{i}(t)\right|^{2}\right)^{\frac{1}{2}} .
$$


Definition 2.1 A resolution $x(t)$ of (1) is an $\omega$ nonperiodic solution, if

$$
\begin{gathered}
x(t+\omega)=-x(t), \quad t \neq t_{k} . \\
x\left(t_{k}+\omega\right)^{+}=-x\left(t_{k}^{+}\right), \quad k=1,2, \cdots,
\end{gathered}
$$

and the smallest positive number $\omega$ is called $\omega$ anti-periodic of function $x(t)$.

Define $P C\left(R^{n}\right)=\left\{x(t)=\left(x_{1}(t), x_{2}(t), \cdots, x_{n}(t)\right)^{T}: R \rightarrow R^{n},\left.x\right|_{\left(t_{k}, t_{k+1}\right]} \in\right.$ $C\left(\left(t_{k}, t_{k+1}\right], R^{n}\right), x\left(t_{k}^{+}\right), x\left(t_{k}\right)$ exist, and $\left.x\left(t_{k}^{-}\right)=x\left(t_{k}\right), k=1,2, \cdots\right\}$. Set $X=\{x$ : $\left.x \in P C\left(R^{n}\right), x(t+\omega)=-x(t), t \in R\right\}$. It is easy to see $X$ is a Banach space with norm $\|x\|=\sup _{t \in[-t, 0]}\left(\sum_{i=1}^{n}\left|x_{i}(t)\right|^{2}\right)^{\frac{1}{2}}$.

Next, It is similar to [13], we have the following lemma.

Lemma 2.1. Let $x(t)=\left(x_{1}(t), x_{2}(t), \cdots, x_{n}(t)\right)^{T}$ be an $\omega$ anti-periodic solution of system (1). For $i=1,2, \cdots, n$, we have

$$
\begin{aligned}
x_{i}(t)= & \int_{t}^{t+\omega} H_{i}(t, s)\left[\sum_{j=1}^{n} d_{i j}(s) f_{j}\left(x_{j}(s)\right)\right. \\
& +\bigwedge_{j=1}^{n} a_{i j}(s) g_{j}\left(x_{j}\left(s-t_{i j}(s)\right)\right)+E_{i}(s) \\
& \left.+\bigvee_{j=1}^{n} b_{i j}(s) g_{j}\left(x_{j}\left(s-t_{i j}(s)\right)\right)\right] d s \\
& +\sum_{t_{k} \in[t, t+\omega]} H_{i}\left(t, t_{k}\right) I_{i k}\left(t_{k}, x_{i}\left(t_{k}\right)\right),
\end{aligned}
$$

where, for $i=1,2, \cdots, n$,

$$
H_{i}(t, s)=-\frac{e^{\int_{t}^{s} a_{i}(\phi) d \phi}}{e^{\int_{0}^{\omega} a_{i}(\phi) d \phi}+1}, s \in[t, t+\omega] .
$$

Lemma 2.2. [15] Let $\Omega$ be a closed convex and nonempty subset of a Banach space $X$. Let $\Pi, \Sigma$ be the operators such that

(i) $\Pi x+\Sigma y \in \Omega$ whenever $x, y \in \Omega$;

(ii) $\Pi$ is compact and continuous;

(iii) $\Sigma$ is a contraction mapping.

Then there exists $z \in \Omega$ such that $z=\Pi z+\Sigma z$.

Lemma 2.3. [13] Let $p, q, t, c_{k}, k=1,2, \cdots$, be constants and $q \geq 0, t>0, c_{k}>0$, and assume that $x(t)$ is piece continuous nonnegative function. Suppose $\Omega$ is a closed 
and nonempty subset of a Banach space $X$. Give $\Pi, \Sigma$ a chance to be the administrators such that

(I) $\Pi x+\Sigma y \in \Omega$ at whatever point $x, y \in \Omega$;

(ii) $\Pi$ is minimal and continuous;

(iii) $\Sigma$ is a compression mapping.

At that point there exists $z \in \Omega$ with the end goal that $z=\Pi z+\Sigma z$.

Lemma 2.4. [13] Let $p, q, t, c_{k}, k=1,2, \cdots$, be constants and $q \geq 0, t>0, c_{k}>0$, and accept that $x(t)$ is piece consistent nonnegative capacity fulfilling

$$
\left\{\begin{array}{l}
D^{+} x(t) \leqslant p x(t)+q \bar{x}(t), t \geqslant t_{0}, t \neq t_{k}, \\
x\left(t_{k}^{+}\right) \leqslant c_{k}\left(x\left(t_{k}\right)\right), k=1,2, \cdots, \\
x(t)=\varphi(t), t \in\left[t_{0}-t, t_{0}\right] .
\end{array}\right.
$$

If there exist $c$ such that for $k=1,2, \cdots$,

$$
\ln c_{k} \leqslant c\left(t_{k}-t_{k-1}\right) \text {. }
$$

and

$$
p+c q+c<0 \text {. }
$$

Then

$$
x(t) \leqslant c \sup _{t \in\left[t_{0}-t, t_{0}\right]}|\varphi(t)| e^{-\lambda\left(t-t_{0}\right)},
$$

where $\bar{x}(t)=\sup _{s \in[t-t, t]} x(s)$,

$$
c=\sup _{1 \leqslant k<+\infty}\left\{e^{c\left(t_{k}-t_{k-1}\right)}, \frac{1}{e^{c\left(t_{k}-t_{k-1}\right)}}\right\},
$$

$\lambda$ is a sole nonnegative resolution of $\lambda+p+c q e^{\lambda t}+c=0$.

Lemma 2.5. [7] Let $u$ and $\Omega$ be two states of system (1), then we have

$$
\left|\bigwedge_{j=1}^{n} a_{i j}(t) g_{j}(u)-\bigwedge_{j=1}^{n} a_{i j}(t) g_{j}(\Omega)\right| \leqslant q \sum_{j=1}^{n}\left|a_{i j}(t)\right|\left|g_{j}(u)-g_{j}(\Omega)\right|,
$$

and

$$
\left|\bigvee_{j=1}^{n} b_{i j}(t) g_{j}(u)-\bigvee_{j=1}^{n} b_{i j}(t) g_{j}(\Omega)\right| \leqslant \sum_{j=1}^{n}\left|b_{i j}(t)\right|\left|g_{j}(u)-g_{j}(\Omega)\right|
$$




\section{Existence and stability of a non-periodic solution}

Here, we derive some sufficient conditions of existence of anti periodic resolution of (1).

Define the operator

$$
\left\{\begin{array}{l}
(\Pi x)(t)=\left(\left(\Pi_{1} x\right)(t),\left(\Pi_{2} x\right)(t), \cdots,\left(\Pi_{n} x\right)(t)\right)^{T}, \\
(\Sigma x)(t)=\left(\left(\Sigma_{1} x\right)(t),\left(\Sigma_{2} x\right)(t), \cdots,\left(\Sigma_{n} x\right)(t)\right)^{T} .
\end{array}\right.
$$

where

$$
\begin{aligned}
\left(\Pi_{i} x\right)(t)= & \int_{t}^{t+\omega} H_{i}(t, s)\left[\sum_{j=1}^{n} d_{i j}(s) f_{j}\left(x_{j}(s)\right)\right. \\
& +\bigwedge_{j=1}^{n} a_{i j}(s) g_{j}\left(x_{j}\left(s-t_{i j}(s)\right)\right) \\
& +\bigvee_{j=1}^{n} b_{i j}(s) g_{j}\left(x_{j}\left(s-t_{i j}(s)\right)\right) \\
& \left.+E_{i}(s)\right] d s
\end{aligned}
$$

$$
\left(\Sigma_{i} x\right)(t)=\sum_{t_{k} \in[t, t+\omega]} H_{i}\left(t, t_{k}\right) I_{i k}\left(t_{k}, x_{i}\left(t_{k}\right)\right), i=1,2, \cdots, n
$$

where $H_{i}(t, s), i=1,2, \ldots, n$, are defined by (4), it is easy to get, for $i=1,2, \cdots, n$,

$$
\frac{1}{1+\chi_{i}} \leqslant\left|H_{i}(t, s)\right| \leqslant \frac{\chi_{i}}{\chi_{i}+1}, s \in[t, t+\omega] .
$$

where $\chi_{i}=e^{\int_{0}^{\omega} a_{i}(\phi) d \phi}$.

Theorem 3.1. Suppose that $(A 1)-(A 4)$ is valid, if the next assumption is satisfied (A5):

$$
\omega\left[\sum_{i=1}^{n}\left(\Upsilon_{i}\right)^{2}\right]^{\frac{1}{2}}+\omega\left[\sum_{i=1}^{n}\left(\Upsilon_{i}^{\prime}\right)^{2}\right]^{\frac{1}{2}}+\sum_{k=1}^{q}\left[\sum_{i=1}^{n}\left(\frac{\chi_{i} c_{i k}}{\chi_{i}+1}\right)^{2}\right]^{\frac{1}{2}}<1
$$

where

$$
\Upsilon_{i}=\frac{\chi_{i}}{\chi_{i}+1}\left[\sum_{j=1}^{n}\left(\bar{d}_{i j} m_{j}\right)^{2}\right]^{\frac{1}{2}}
$$




$$
\Upsilon_{i}^{\prime}=\frac{\chi_{i}}{\chi_{i}+1}\left[\sum_{j=1}^{n}\left(\left(\bar{a}_{i j}+\bar{b}_{i j}\right) n_{j}\right)^{2}\right]^{\frac{1}{2}},
$$

then (1) has a unique $\omega$ nonperiodic solution.

Theorem 3.2. Assume that (A1)-(A4) hold, if the following assumption is satisfied

$$
\sum_{k=1}^{q}\left[\sum_{i=1}^{n}\left(\frac{\chi_{i} c_{i k}}{\chi_{i}+1}\right)^{2}\right]^{\frac{1}{2}}<1
$$

it is valid that (1.1) possesses more than $\omega$ nonperiodic resolutions.

Proof. We define the operator $\Pi, \Sigma$ as (8). Choosing

$$
\rho \geqslant \frac{\left(n \omega \bar{d} M_{f}+n \omega(\bar{a}+\bar{b}) M_{g}+\omega \bar{E}+q \bar{I}\right)\left[\sum_{i=1}^{n}\left(\frac{\chi_{i}}{\chi_{i}+1}\right)^{2}\right]^{\frac{1}{2}}}{1-\sum_{k=1}^{q}\left[\sum_{i=1}^{n}\left(\frac{\chi_{i} c_{i k}}{\chi_{i}+1}\right)^{2}\right]^{\frac{1}{2}}}>0
$$

For $x, y \in B_{\rho}=\{x \in X:\|x\| \leqslant \rho\}$, we get

$$
\begin{aligned}
& \|(\Pi x)(t)+(\Sigma y)(t)\| \\
= & \sup _{t \in[0, \omega]}\left\{\sum_{i=1}^{n} \mid \int_{t}^{t+\omega} H_{i}(t, s)\left[\sum_{j=1}^{n} d_{i j}(s) f_{j}\left(x_{j}(s)\right)+\bigwedge_{j=1}^{n} a_{i j}(s) g_{j}\left(x_{j}\left(s-t_{i j}(s)\right)\right)\right.\right. \\
& \left.+\bigvee_{j=1}^{n} b_{i j}(s) g_{j}\left(x_{j}\left(s-t_{i j}(s)\right)\right)+E_{i}(s)\right] d s \\
& +\left.\sum_{t_{k} \in[t, t+\omega]} H_{i}\left(t, t_{k}\right) I_{i k}\left(t_{k}, y_{i}\left(t_{k}\right)\right)\right|^{2}
\end{aligned}
$$




$$
\begin{aligned}
& =\sup _{t \in[0, \omega]}\left\{\sum_{i=1}^{n} \mid \int_{t}^{t+\omega} H_{i}(t, s)\right. \\
& \times\left[\sum_{j=1}^{n} d_{i j}(s) f_{j}\left(x_{j}(s)\right)-\sum_{j=1}^{n} d_{i j}(s) f_{j}(0)\right. \\
& +\bigwedge_{j=1}^{n} a_{i j}(s) g_{j}\left(x_{j}\left(s-t_{i j}(s)\right)\right)-\bigwedge_{j=1}^{n} a_{i j}(s) g_{j}(0) \\
& \left.+\bigvee_{j=1}^{n} b_{i j}(s) g_{j}\left(x_{j}\left(s-t_{i j}(s)\right)\right)-\bigvee_{j=1}^{n} b_{i j}(s) g_{j}(0)+E_{i}(s)\right] d s \\
& \left.+\left.\sum_{t_{k} \in[t, t+\omega]} H_{i}\left(t, t_{k}\right) I_{i k}\left(t_{k}, y_{i}\left(t_{k}\right)\right)\right|^{2}\right\}^{\frac{1}{2}} \\
& \leqslant\left[\sum_{i=1}^{n}\left(\frac{\chi_{i}}{\chi_{i}+1} \int_{0}^{\omega} \sum_{j=1}^{n} \bar{d}_{i j}\left|f_{j}\left(x_{j}(s)\right)\right| d s \mid\right)^{2}\right]^{\frac{1}{2}}+\left[\sum_{i=1}^{n}\left(\frac{\chi_{i}}{\chi_{i}+1} \int_{0}^{\omega} \sum_{j=1}^{n} \overline{(a}_{i j}+\bar{b}_{i j}\right)\right. \\
& \left.\left.\times\left|g_{j}\left(x_{j}\left(s-t_{i j}(s)\right)\right)\right| d s \mid\right)^{2}\right]^{\frac{1}{2}}+\omega \bar{E}\left[\sum_{i=1}^{n}\left(\frac{\chi_{i}}{\chi_{i}+1}\right)^{2}\right]^{\frac{1}{2}} \\
& +\left[\sum_{i=1}^{n}\left(\frac{\chi_{i}}{\chi_{i}+1} \sum_{k=1}^{q}\left|I_{i k}\left(t_{k}, y_{i}\left(t_{k}\right)\right)-I_{i k}\left(t_{k}, 0\right)\right|\right)^{2}\right]^{\frac{1}{2}} \\
& +\left[\sum_{i=1}^{n}\left(\frac{\chi_{i}}{\chi_{i}+1} \sum_{k=1}^{q}\left|I_{i k}\left(t_{k}, 0\right)\right|\right)^{2}\right]^{\frac{1}{2}} \\
& \leqslant\left\{\sum_{k=1}^{q}\left[\sum_{i=1}^{n}\left(\frac{\chi_{i} c_{i k}}{\chi_{i}+1}\right)^{2}\right]^{\frac{1}{2}}\right\} \rho \\
& +\left(n \omega \bar{d} M_{f}+n \omega(\bar{a}+\bar{b}) M_{g}+\omega \bar{E}+q \bar{I}\right) \\
& \times\left[\sum_{i=1}^{n}\left(\frac{\chi_{i}}{\chi_{i}+1}\right)^{2}\right]^{\frac{1}{2}} \\
& \leqslant \rho
\end{aligned}
$$

Therefore, $\Pi x+\Sigma y \in B_{\rho}$. Since $f_{j}(\cdot), g_{j}(\cdot), j=1,2, \cdots, n$, are continuous. Thus the operator $\Pi$ is continuous. For $x \in B_{\rho}$, we have

$$
\|\Pi x\| \leqslant\left(n \omega \bar{d} M_{f}+n \omega(\bar{a}+\bar{b}) M_{g}+\omega \bar{E}\right)\left[\sum_{i=1}^{n}\left(\frac{\chi_{i}}{\chi_{i}+1}\right)^{2}\right]^{\frac{1}{2}}
$$


i.e. $\Pi$ is uniformly surrounded on $B_{\rho}$. Then, let us show the accuracy of $\Pi$. For $t_{1}, t_{2} \in[0, \omega]$, it is valid that

$$
\begin{aligned}
& \left\|(\Pi x)\left(t_{1}\right)-(\Pi x)\left(t_{2}\right)\right\| \\
\leqslant & {\left[\sum_{i=1}^{n}\left|\int_{0}^{\omega}\right| H_{i}\left(t_{1}, s\right)-H_{i}\left(t_{2}, s\right) \mid\left[\sum_{j=1}^{n} d_{i j}(s) f_{j}\left(x_{j}(s)\right)\right.\right.} \\
& +\bigwedge_{j=1}^{n} a_{i j}(s) g_{j}\left(x_{j}\left(s-t_{i j}(s)\right)\right) \\
& \left.\left.+\bigvee_{j=1}^{n} a_{i j}(s) g_{j}\left(x_{j}\left(s-t_{i j}(s)\right)\right)+E_{i}(s)\right]\left.d s\right|^{2}\right]^{\frac{1}{2}} \\
\leqslant & \sum_{i=1}^{n} \frac{1}{\chi_{i}+1} \int_{0}^{\omega} \mid e^{\int_{t_{1}}^{s} a_{i}(\phi) d \phi}-e^{\int_{t_{2}}^{s} a_{i}(\phi) d \phi \mid} \\
& \times\left[\sum_{j=1}^{n} \bar{d}_{i j} M_{f}+\bigwedge_{j=1}^{n} \bar{a}_{i j} M_{g}+\bigvee_{j=1}^{n} \bar{b}_{i j} M_{g}+\bar{E}\right] d s \\
\leqslant & \left|t_{1}-t_{2}\right|\left[\sum_{j=1}^{n} \bar{d}_{i j} M_{f}+\bigwedge_{j=1}^{n} \bar{a}_{i j} M_{g}\right. \\
& \left.+\bigvee_{j=1}^{n} \bar{b}_{i j} M_{g}+\bar{E}\right] \omega a^{+} \sum_{i=1}^{n} \frac{\chi_{i}}{\chi_{i}+1} \\
\leqslant & \left|t_{1}-t_{2}\right|\left[n \bar{d} M_{f}+n(\bar{a}+\bar{b}) M_{g}+\bar{E}\right] \omega a^{+} \sum_{i=1}^{n} \frac{\chi_{i}}{\chi_{i}+1}
\end{aligned}
$$

Consequently, by methods for Arzela-Ascoli hypothesis, $\Pi$ is reduced on $B_{\rho}$. By presumption (A6), plainly $\Sigma$ is constriction mapping. Utilizing Lemma 2.2, framework (1) has in any event $\omega$ against occasional arrangement.

Assume that $x^{*}(t)=\left(x_{1}^{*}(t),, \cdots, x_{n}^{*}(t)\right)^{T}$ is an $\omega$-occasional arrangement of framework (1). In this area, we will develop some appropriate Lyapunov practical to demonstrate the worldwide exponential security of this enemy of occasional arrangement.

Theorem 3.3. Suppose that assumptions $(A 1)-(A 5)$ hold. If the following assumptions are satisfied

(A7) there exist $c, \bar{c}_{i k} \geq 0, i=1,2, \cdots, n, k=1,2, \cdots$, such that

$$
\left|u+I_{i k}(t, u)-\Omega-I_{i k}(t, \Omega)\right| \leqslant \bar{c}_{i k}|u-\Omega|, t \in[0, \omega], u, \Omega \in R,
$$

and for $k=1,2, \cdots$,

$$
2 \ln c_{k} \leqslant c\left(t_{k}-t_{k-1}\right)
$$


(A8) there exist $c_{i}>0$ and $\delta_{i j}, \eta_{i j}, \vartheta_{i j}, \xi_{i j} \in R, i=1,2, \cdots, n$ such that

$$
-\Theta_{1}+c \Theta_{2}+c=0
$$

where

$$
\begin{gathered}
\Theta_{1}=\min _{1 \leqslant i \leqslant n}\left\{2 a_{i}^{-}-\sum_{j=1}^{n}\left(\bar{d}_{i j}\right)^{2 \delta_{i j}} m_{j}^{2 \eta_{i j}}\right. \\
-\sum_{j=1}^{n} \frac{c_{j}}{c_{i}}\left(\bar{d}_{j i}\right)^{2\left(1-\delta_{i j}\right)} m_{j}^{2\left(1-\eta_{i j}\right)} \\
\left.-\sum_{j=1}^{n}\left(\bar{a}_{i j}+\bar{b}_{i j}\right)^{2 \vartheta_{i j}} n_{j}^{2 \xi_{i j}}\right\} . \\
\Theta_{2}=\max _{1 \leqslant i \leqslant n}\left\{\sum_{j=1}^{n} \frac{c_{j}}{c_{i}}\left(\bar{a}_{j i}+\bar{b}_{j i}\right)^{2\left(1-\vartheta_{i j}\right)} m_{j}^{2\left(1-\xi_{i j}\right)}\right\}, \\
c_{k}=\max _{1 \leqslant i \leqslant n}\left\{\bar{c}_{i k}\right\}, \\
c=\max _{1 \leqslant k<+\infty}\left\{e^{c\left(t_{k}-t_{k-1}\right)}, \frac{1}{e^{c\left(t_{k}-t_{k-1}\right)}}\right\}
\end{gathered}
$$

then $\omega$ anti periodic solution of system (1) is globally exponentially stable with convergence rate $\lambda / 2$, and $\lambda$ is an unique positive solution of $\lambda-\Theta_{1}+c \Theta_{2} e^{\lambda t}+c=0$.

Proof. Suppose $x^{*}(t)=\left(x_{1}^{*}(t), x_{2}^{*}(t), \cdots, x_{n}^{*}(t)\right)^{T}$ is an $\omega$ nonperiodic arrangement of (1). $x(t)=\left(x_{1}(t), x_{2}(t), \cdots, x_{n}(t)\right)^{T}$ is an arrangement of $(1)$. Set $y(t)=$ $x(t)-x^{*}(t)$. Then, for $k=1,2, \cdots, i=1,2, \cdots, n$,

$$
\left\{\begin{aligned}
y_{i}^{\prime}(t)= & -a_{i}(t)\left(x_{i}(t)-x_{i}^{*}(t)\right) \\
& +\sum_{j=1}^{n} d_{i j}(t)\left[f_{j}\left(x_{j}(t)\right)-f_{j}\left(x_{j}^{*}(t)\right)\right] \\
& +\bigwedge_{j=1}^{n} a_{i j}(t) g_{j}\left(x_{j}\left(t-t_{i j}(t)\right)\right) \\
& -\bigwedge_{j=1}^{n} a_{i j}(t) g_{j}\left(x_{j}^{*}\left(t-t_{i j}(t)\right)\right) \\
& +\bigvee_{j=1}^{n} b_{i j}(t) g_{j}\left(x_{j}\left(t-t_{i j}(t)\right)\right) \\
& -\bigvee_{j=1}^{n} b_{i j}(t) g_{j}\left(x_{j}^{*}\left(t-t_{i j}(t)\right)\right), t \geq 0, t \neq t_{k} \\
y_{i}\left(t_{k}^{+}\right)= & x_{i}\left(t_{k}\right)-x_{i}^{*}\left(t_{k}\right)+I_{i k}\left(t_{k}, x_{i}\left(t_{k}\right)\right) \\
& -I_{i k}\left(t_{k}, x_{i}^{*}\left(t_{k}\right)\right) .
\end{aligned}\right.
$$


Considering the following function

$$
\Omega(t)=\sum_{i=1}^{n} c_{i}\left|y_{i}(t)\right|^{2} .
$$

Computing the above right derivative of $\Omega(t)$, for $t \neq t_{k}$,

$$
\begin{aligned}
& D^{+} \Omega(t) \\
= & \sum_{i=1}^{n} 2 c_{i} D^{+}\left|y_{i}(t)\right| \\
\leqslant & \sum_{i=1}^{n}-2 c_{i} a_{i}(t)\left|y_{i}(t)\right|\left|y_{i}(t)\right| \\
& +\sum_{i=1}^{n} 2 c_{i} \sum_{j=1}^{n}\left|d_{i j}(t)\right|\left|y_{i}(t)\right|\left|f_{j}\left(x_{j}(t)\right)-f_{j}\left(x_{j}^{*}(t)\right)\right| \\
& +\sum_{i=1}^{n} 2 c_{i} \sum_{j=1}^{n}\left(\left|a_{i j}(t)\right|+\left|b_{i j}(t)\right|\right)\left|y_{i}(t)\right| \\
& \times\left|g_{j}\left(x_{j}\left(t-t_{i j}(t)\right)\right)-g_{j}\left(x_{j}^{*}\left(t-t_{i j}(t)\right)\right)\right| \\
\leqslant & \sum_{i=1}^{n}-2 c_{i} a_{i}^{-}\left|y_{i}(t)\right|^{2}+\sum_{i=1}^{n} 2 c_{i} \sum_{j=1}^{n} \bar{d}_{i j}\left|y_{i}(t)\right| m_{j}\left|y_{j}(t)\right| \\
& +\sum_{i=1}^{n} 2 c_{i} \sum_{j=1}^{n}\left(\bar{a}_{i j}+\bar{b}_{i j}\right)\left|y_{i}(t)\right| n_{j}\left|y_{j}\left(t-t_{i j}(t)\right)\right|
\end{aligned}
$$

Using inequality $a b \leqslant \frac{1}{2} a^{2}+\frac{1}{2} b^{2}$, we have

$$
\begin{aligned}
& \sum_{j=1}^{n} \bar{d}_{i j}\left|y_{i}(t)\right| m_{j}\left|y_{j}(t)\right| \\
= & \sum_{j=1}^{n}\left[\left(\bar{d}_{i j}\right)^{\delta_{i j}} m_{j}^{\eta_{i j}}\left|y_{i}(t)\right|\right]\left[\left(\bar{d}_{i j}\right)^{1-\delta_{i j}} m_{j}^{1-\eta_{i j}}\left|y_{j}(t)\right|\right] \\
\leqslant & \sum_{j=1}^{n}\left[\frac{1}{2}\left(\bar{d}_{i j}\right)^{2 \delta_{i j}} m_{j}^{2 \eta_{i j}}\left|y_{i}(t)\right|^{2}\right. \\
& \left.+\frac{1}{2}\left(\bar{d}_{i j}\right)^{2\left(1-\delta_{i j}\right)} m_{j}^{2\left(1-\eta_{i j}\right)}\left|y_{j}(t)\right|^{2}\right]
\end{aligned}
$$


and

$$
\begin{aligned}
& \sum_{j=1}^{n}\left(\bar{a}_{i j}+\bar{b}_{i j}\right)\left|y_{i}(t)\right| n_{j}\left|y_{j}\left(t-t_{i j}(t)\right)\right| \\
\leqslant & \sum_{j=1}^{n}\left[\frac{1}{2}\left(\bar{a}_{i j}+\bar{b}_{i j}\right)^{2 \vartheta_{i j}} n_{j}^{2 \xi_{i j}}\left|y_{i}(t)\right|^{2}\right. \\
& +\frac{1}{2}\left(\bar{a}_{i j}+\bar{b}_{i j}\right)^{2\left(1-\vartheta_{i j}\right)} m_{j}^{2\left(1-\xi_{i j}\right)} \\
& \left.\times\left|y_{j}\left(t-t_{i j}(t)\right)\right|^{2}\right]
\end{aligned}
$$

Substituting (21) and (22) into (20), we have, for $t \neq t_{k}$,

$$
\begin{aligned}
& D^{+} \Omega(t) \\
\leqslant & \sum_{i=1}^{n} c_{i}\left\{-2 a_{i}^{-}\left|y_{i}(t)\right|+\sum_{j=1}^{n}\left[\left(\bar{d}_{i j}\right)^{2 \delta_{i j}} m_{j}^{2 \eta_{i j}}\left|y_{i}(t)\right|^{2}\right.\right. \\
& \left.+\left(\bar{d}_{i j}\right)^{2\left(1-\delta_{i j}\right)} m_{j}^{2\left(1-\eta_{i j}\right)}\left|y_{j}(t)\right|^{2}\right] \\
& +\sum_{j=1}^{n}\left[\left(\bar{a}_{i j}+\bar{b}_{i j}\right)^{2 \vartheta_{i j}} n_{j}^{2 \xi_{i j}}\left|y_{i}(t)\right|^{2}\right. \\
& \left.\left.+\left(\bar{a}_{i j}+\bar{b}_{i j}\right)^{2\left(1-\vartheta_{i j}\right)} m_{j}^{2\left(1-\xi_{i j}\right)}\left|y_{j}\left(t-t_{i j}(t)\right)\right|^{2}\right]\right\} \\
= & \sum_{i=1}^{n} c_{i}\left\{\left[-2 a_{i}^{-}+\sum_{j=1}^{n}\left(\bar{d}_{i j}\right)^{2 \delta_{i j}} m_{j}^{2 \eta_{i j}}\right.\right. \\
& +\sum_{j=1}^{n} \frac{c_{j}}{c_{i}}\left(\bar{d}_{j i}\right)^{2\left(1-\delta_{i j}\right)} m_{j}^{2\left(1-\eta_{i j}\right)} \\
& \left.+\sum_{j=1}^{n}\left(\bar{a}_{i j}+\bar{b}_{i j}\right)^{2 \vartheta_{i j}} n_{j}^{2 \xi_{i j}}\right]\left|y_{i}(t)\right|^{2} \\
& +\sum_{j=1}^{n} \frac{c_{j}}{c_{i}}\left(\bar{a}_{j i}+\bar{b}_{j i}\right)^{2\left(1-\vartheta_{i j}\right)} m_{j}^{2\left(1-\xi_{i j}\right)} \\
& \left.\times\left|y_{j}\left(t-t_{i j}(t)\right)\right|^{2}\right\} \\
\leqslant & -\Theta_{1} \Omega(t)+\Theta_{2} \bar{\Omega}(t)
\end{aligned}
$$

where $\Omega(t)=\sup _{t-t \leqslant \eta \leqslant t} \Omega(\eta)$. From (A6), we have

$$
\Omega\left(t_{k}^{+}\right)=\sum_{i=1}^{n} c_{i}\left|y_{i}\left(t_{k}^{+}\right)\right|^{2} \leqslant \sum_{i=1}^{n} c_{i} \bar{c}_{i k}^{2}\left|y_{i}\left(t_{k}\right)\right|^{2}<c_{k}^{2} \Omega\left(t_{k}\right) .
$$


From Lemma 2.3, there is $c>1$ satisfying

$$
\Omega(t) \leqslant c\left(\sup _{-t \leqslant t \leqslant 0} \Omega(t)\right) e^{-\lambda t}
$$

Thus

$$
\left\|x(t)-x^{*}(t)\right\| \leqslant\left(\frac{c \max _{1 \leqslant i \leqslant n}\left(c_{i}\right)}{\min _{1 \leqslant i \leqslant n}\left(c_{i}\right)}\right)^{\frac{1}{2}}\left\|\varphi-\varphi^{*}\right\| e^{-\lambda t / 2} .
$$

The validity of the theorem is completed.

The global exponential stability of FCNNs is important dynamical behavior. Time delays and impulsive effects often cause system instability or oscillatory behaviour. It is clear that the results obtained are related with the time delay and impulses for justifying global exponentially stability of $\omega$ anti periodic solution of system (1).

\section{A numerical example}

In this segment, a precedent is given to demonstrate adequacy of results acquired.

Example 5.1 Consider the accompanying FCNNs with time-changing deferral and hasty impacts.

$$
\left\{\begin{aligned}
x_{i}^{\prime}(t)= & -a_{i}(t) x_{i}(t)+\sum_{j=1}^{2} d_{i j}(t) f_{j}\left(x_{j}(t)\right) \\
& +\bigwedge_{j=1}^{2} a_{i j}(t) g_{j}\left(x_{j}\left(t-t_{i j}(t)\right)\right) \\
& +\bigvee_{j=1}^{2} b_{i j}(t) g_{j}\left(x_{j}\left(t-t_{i j}(t)\right)\right) \\
& +E_{i}(t), t \neq \frac{k \pi}{2}, k=1,2, \cdots, \\
\left.\Delta x_{i}\left(t_{k}\right)\right)= & -\frac{2}{3} x_{i}\left(t_{k}\right), t=t_{k}=\frac{k \pi}{2}, i=1,2,
\end{aligned}\right.
$$

where $a_{1}(t)=a_{2}(t)=\frac{1}{8}, f_{j}(x)=g_{j}(x)=\arctan x(j=1,2)$.

$$
\begin{aligned}
\left(d_{i j}(t)\right)_{2 \times 2} & =\left(\begin{array}{cc}
1 / 4 & 1 / 8 \\
1 / 6 & 1 / 3
\end{array}\right), \\
\left(a_{i j}(t)\right)_{2 \times 2} & =\left(\begin{array}{cc}
1 / 8 & 1 / 6 \\
1 / 6 & 1 / 8
\end{array}\right), \\
\left(b_{i j}(t)\right)_{2 \times 2} & =\left(\begin{array}{cc}
1 / 16 & 1 / 4 \\
1 / 4 & 1 / 16
\end{array}\right), \\
\left(E_{i}(t)\right)_{2 \times 1} & =\left(\begin{array}{c}
1 / 4 \sin t \\
1 / 3 \cos t
\end{array}\right) .
\end{aligned}
$$


impulsive functions $I_{1 k}(t, x)=I_{2 k}(t, x)=-\frac{2}{3} x$, impulsive points $t_{k}=\frac{k \pi}{2}, t_{11}(t)=$ $t_{21}(t)=|\sin (2 \pi t)|, t_{12}(t)=t_{22}(t)=|\cos (2 \pi t)|$, then, we can easily check that $u=\Omega=\frac{\pi}{2}, c_{1 k}=c_{2 k}=\frac{2}{3}, \bar{c}_{1 k}=\bar{c}_{2 k}=\frac{1}{3}, c_{k}=\frac{1}{3}, c=1, c_{1}=c_{2}=e^{\frac{\pi}{8}}$, Taking $\delta_{i j}=\eta_{i j}=\vartheta_{i j}=\xi_{i j}=\frac{1}{2}(i=1,2), \frac{2 \ln c_{k}}{t_{k}-t_{k-1}} \leqslant-1.39=c$.

It is easy to conclude that assumptions (A6) and (A8) hold true. Numerical arrangement $x(t)=\left(x_{1}(t), x_{2}(t)\right)^{T}$ of frameworks (27) for introductory esteem $\varphi(s)=(0.5,-0.4)^{T}, s \in[-2,0]$.

\section{Conclusion}

In this paper, the presence and internationally exponential solidness of the counter intermittent answer for fuzzy cell neural systems with time-differing delays are considered. Some adequate conditions set up here are effortlessly confirmed what's more, these conditions are related with parameters of the framework (1). The acquired criteria can be connected to plan all around exponential stable of hostile to occasional ceaseless fuzzy cell neural systems.

\section{R E F E R E N C E S}

1. L. O. ChuA, And L. YAng, "Cellular neural networks:Application", IEEE Trans. Circ. Syst.I, 35, pp. 1273-1290, 1988.

2. A. Chen, And J. CAO, "Existence and attractivity of almost periodic solutions for cellular neural networks with distributed delays and variable coefficients", Appl. Math. Comput., 134, pp. 125-140, 2003.

3. C. HuAng, And J. CAO, "Almost sure exponential stability of stochastic cellular neural networks with unbounded distributed delays", Neurocomputing, 72, pp. 33523356, 2009.

4. Y. Xia, J. CaO, and S. Cheng, "Global exponential stability of delayed cellular neural networks with impulses", Neurocomputing, 70, pp. 2495-2501, 2007.

5. Y. YANG, AND J. CAO, "Stability and periodicity in delayed cellular neural networks with impulsive effects", Nonolinear Analysis:RWA, 8, pp. 362-374,2007.

6. Y. LI, AND J. WANG, "An analysis on the global exponential stability and the existence of periodic solutions for non-autonomous hybird BAM neural networks with distributed delays and impulsies", Comput. Math. Appl., 56, pp. 2256-2267,2008.

7. T. YANG, AND L. YANG, "The global stability of fuzzy cellular neural networks", IEEE Trans. Circ. Syst. I, 43, pp. 880-883, 1996.

8. X. Li, R. Rakkiyappan, and P. Balasubramaniam, "Existence and global stability analysis of equilibrium of fuzzy cellular neural networks with time delay in the leakage term under impulsive perturbations", Journal of the Franklin Institute, 348,pp. 135155,2011 .

9. Q. ZhANG, AND R. XIANG, "Global asymptotic stability of fuzzy cellular neural networks with time-varying delays", Phy. Lett. A, 372, pp. 3971-3977, 2008. 
10. W. HE, AND L. CHu, "Exponential stability criteria for fuzzy bidirectional associative memory Cohen-Grossberg neural networks with mixed delays and impulses", Advances Difference Equations, (2017): 61, 2017.

11. G. YANG, "New results on the stability of fuzzy cellular neural networks with timevarying leakage delays", Neural Computing and Applications, 25, pp. 1709-1715, 2014.

12. J. SнAо, "An anti-periodic solution for a class of recurrent neural networks", J. Comput. Appl. Math., 228, pp. 231-237, 2009.

13. L. PAN, AND J. CAO, "Anti-periodic solution for delayed cellular neural networks with impulsive effects", Nonlinear Analysis: Real World Applications, 12, pp. 30143027, 2011.

14. A. Abdurahman, And H. JiAng, "The existence and stability of the anti-periodic solution for delayed Cohen-Grossberg neural networks with impulsive effects", Neurocomputing 149, pp.22-28, 2015.

15. M.A. Krasnoselskir, Positive solutions of operator equations, Groningen, Netherlands, 1964 .

Ivan P. Stanimirović

Faculty of Science and Mathematics

Department of Computer Science

18000 Nis, Serbia

ivcastanimirovic@gmail.com 